\title{
A study on Quark-Gluon plasma equation of state using thermal quark mass
}

\author{
Yogesh Kumar ${ }^{1, a}$ and S. Somorendro Singh ${ }^{2}$ \\ ${ }^{1}$ Department of Physics, Deshbandhu College, University of Delhi, Kalkaji, New Delhi-110019, India \\ ${ }^{2}$ Department of Physics and Astrophysics, University of Delhi, New Delhi-110007, India
}

\begin{abstract}
We study the QGP equation of state (EoS) using a simple statistical model. In this, temperature dependent quark mass is used with the inclusion of curvature term. The EoS such as pressure, energy density, entropy and speed of sound at zero chemical potential are evaluated. The model results provide QGP EoS which are in good agreement with our earlier work and the lattice QCD data.
\end{abstract}

\section{Introduction}

It is widely accepted that the ulta-relativistic heavy-ion collisions bring us information about the properties of QGP [1]. At high temperature and/or high chemical potential, quantum chromo-dynamics (QCD) predicts the order of phase transition from hadronic phase to the quark-gluon plasma phase. Such phase transition form a new state of matter, called the quark-gluon plasma (QGP) [2-5].

Even after a lot of experimental as well as theoretical research, our knowledge regarding the properties of QGP is still not good [6,7]. Moreover, theoretical works to study the formation of a QGP in high-energy heavy-ion collisions require an equation of state. For several decades, the bag model EoS has been used to describe the QGP $[8,9]$.

Many theoretician believe that the zero value of chemical potential is a valid approximation for the formation of a QGP in nucleus-nucleus collisions at the BNL (RHIC) and CERN (LHC). It is also noticed that the most lattice QCD calculations for the QGP EoS correspond to zero or very small values of the chemical potential. Therefore, in this present work, we introduce a simple phenomenological model as a quasi particle model which might be a better complement to the lattice QCD simulations in the exploration of QCD thermodynamics for producing the QGP EoS with the help of thermal quark mass incorporating curvature term.

Thus, we organize the paper as: In section 2, we give the description of model. In section 3, we present the QGP equation of state. In section 4, the results are presented and finally in the last section 5 , we give the conclusion .

\section{Description of model}

The search for quark gluon plasma has become an interesting area in high energy heavy ion collisions. Several theoretical models have been proposed in order to make the theory thermodynamically consistent as well as compatible with perturbative and non-perturbative calculations of QCD [10, 11].

\footnotetext{
ae-mail: yogesh.du81@gmail.com
} 
In past decade, it was considered that QGP is made up of quasi-particles with a temperature dependent mass $[12,13]$. A simple model is used in order to derive the EoS of QGP using thermal quark mass and incorporating curvature term in the density of states at zero chemical potential.

In this work, we consider QGP as a system of quasi-particles contain quarks and gluons. These particles are temperature dependent and their masses are generated due to interactions among quarks and gluons in the hot and dense medium [14-17]. Therefore, the quasi-particle model in QCD is a phenomenological model whose masses are temperature dependent called thermal quark mass. This model is thermodynamically self consistent by incorporating suitable parametrization factor in order to match Lattice QCD results.

So far we have calculated the free energy with effect of curvature term using dynamical quark mass [18]. We now extend the previous work using finite quark mass in order to calculate QGP EoS [19]. The finite quark mass is temperature dependent and defined as [19]:

$$
m_{q}^{2}(T)=\gamma_{q} g^{2}(k) T^{2},
$$

where, $g^{2}(k)=4 \pi \alpha_{s}$ with QCD strong coupling constant $\alpha_{s}$ defined as [18, 19];

$$
\alpha_{s}=\frac{4}{\left(33-2 N_{f}\right) \ln \left(1+\frac{k^{2}}{\Lambda^{2}}\right)},
$$

and,

$$
k=\left[\frac{\gamma N^{\frac{1}{3}} T^{2} \Lambda^{2}}{2}\right]^{\frac{1}{4}} .
$$

The number of flavor is taken as $N_{f}=3$. The parametrization factor $\gamma^{2}=2\left[\frac{1}{\gamma_{q}^{2}}+\frac{1}{\gamma_{g}^{2}}\right]$ is used with $\gamma_{q}=1 / 6[19]$ and $\gamma_{g}=0.02 \gamma_{q}$ is used to fit into Lattice QCD results.

\section{QGP equation of state}

The free energy, $F_{i}$ for quarks, gluons and interface is modified with thermal quark mass including curvature term using Ref. [18, 19]. It is defined as:

$$
F_{i}=\mp T g_{i} \int d k \rho_{i}(k) \ln \left(1 \pm e^{-\left(\sqrt{m_{i}^{2}+k^{2}}\right) / T}\right),
$$

where $\rho_{i}(k)$ is the density of states of the particular particle $i$ (quarks, gluons and interface), and $g_{i}$ is the degeneracy factor and its value is taken from Ref. [20]. The interfacial free energy is defined as:

$$
F_{\text {interface }}=\frac{1}{4} R^{2} T^{3} \gamma .
$$

where, $R$ is the radius of QGP droplet. Using above equation, we can thus compute the total free energy $F_{\text {total }}$ as,

$$
F_{\text {total }}=F_{q=u, d, s}+F_{\text {gluon }}+F_{\text {interface }} .
$$

From above total energies, we can explain free energy evolution of quark gluon plasma in which the curvature term with finite quark mass indicate the propensity of the system for QGP droplet formation.

From above equation, we can calculate pressure and energy density using thermodynamic relation. It is given as Ref. [20]:

$$
P=-\frac{d}{d v} F_{\text {total }} .
$$


where, i stands for quarks, gluons and interface term. Total pressure is the sum of the pressure due to all the constituents. Further the energy density is used as [20],

$$
\varepsilon=T \frac{d}{d T} P-P
$$
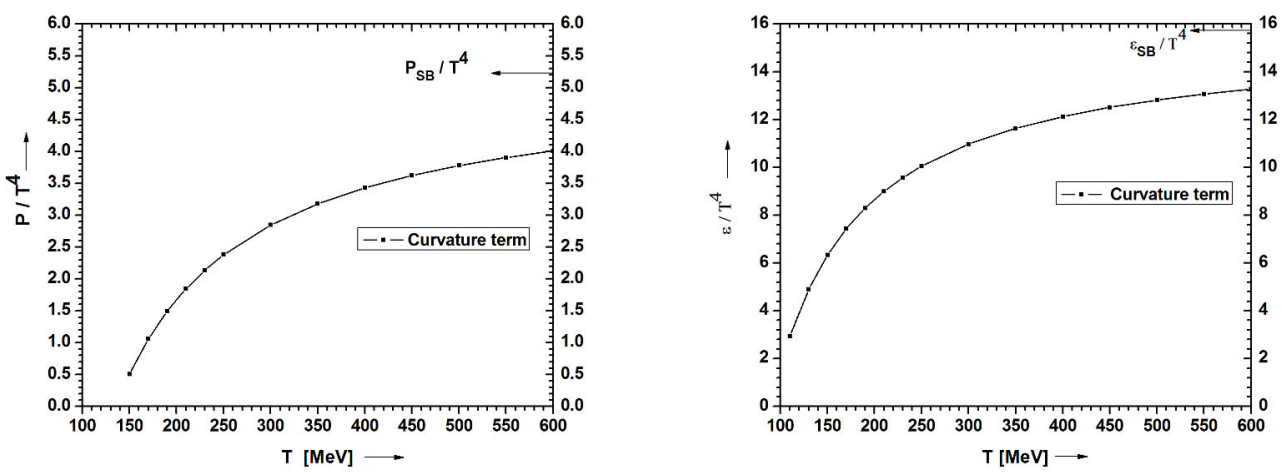

Figure 1. The variation of $P / T^{4}$ on L.H.S. and $\varepsilon / T^{4}$ on R.H.S. with temperature (T) is shown with quark mass including curvature term

We adjust the parameters of the model accordingly. Further we compare their predictions for energy density and pressure of the QGP with those obtained from free energy evolution. Using these relations, we calculate $P / T^{4}$ and $\varepsilon / T^{4}$. These relations are useful to study the quark gluon plasma equation of state using temperature dependent quark mass with curvature term. We can also compute entropy and speed of sound using above thermodynamical relation. Finally our findings are very much similar to our earlier work and also with Lattice results.

\section{Results}

In order to produce the QGP EoS using a simple model, we show the plots of $P / T^{4}$ and $\varepsilon / T^{4}$ with temperature (T) in figure [1]. Both the output of graphs are almost same as our earlier results [20] and also similar to Lattice results especially at high temperature $\mathrm{T} \sim 600 \mathrm{MeV}$.

Similarly in figure [2], entropy $S / T^{3}$ and speed of sound with respect to temperature (T) are shown which are in good agreement with earlier work. At such high temperature, pressure, energy density, entropy and speed of sound approach a value which is below the Stefan-Boltzmann (SB) limit in both figures.

Here the thermal quark mass with the inclusion of curvature term is nicely fitted in the calculation of EoS and consistent with earlier work. It is again observed that there is a negligibly small increment in the QGP EoS which in turn verify that the QGP EoS are not invalidate the calculations by introducing the thermal correction in quark mass. Therefore our results using thermal quark mass with the effect of curvature term are almost same as Ref. [20].

Finally we show that the theoretical model with the thermal correction in the quark mass including curvature term does not alter the results of Gosain et al. very much, in fact the change caused in the 

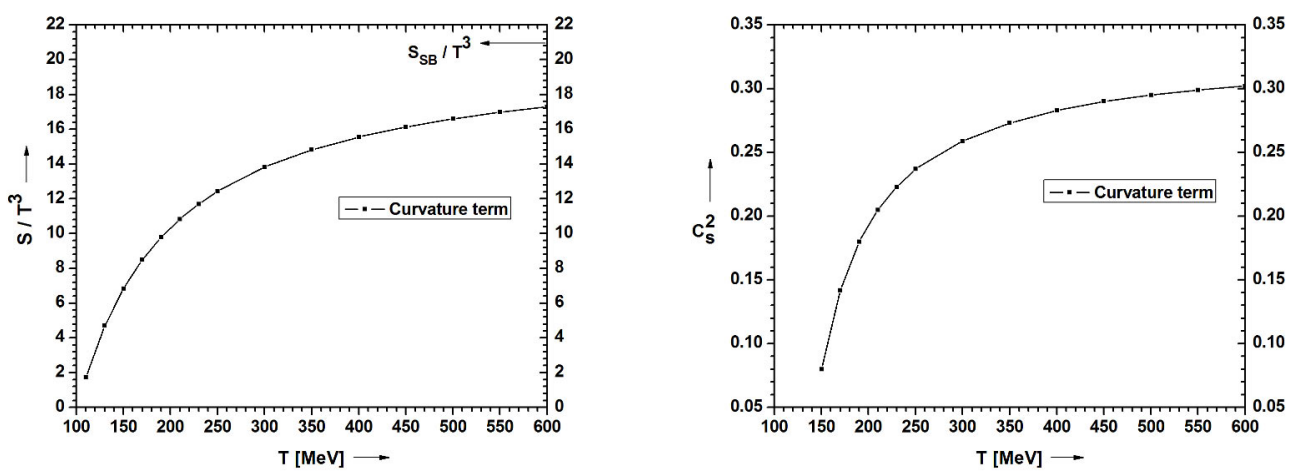

Figure 2. The variation of $S / T^{3}$ on L.H.S. and $C_{s}^{2}$ on R.H.S. with temperature (T) is shown with quark mass including curvature term

QGP EoS is negligible. Our results are very much similar with the results of Ref. [20]. Also the EoS prediction by other models are in conformity with lattice results $[1,2,14,16]$.

\section{Conclusion}

Overall, the present model gives a negligibly small improvement so it varifies the previous theoretical results in QGP EoS. Thus, the thermal quark mass with the inclusion of curvature term shows the useful information to study QGP EoS in high energy heavy-ion collisions.

\section{References}

[1] P. K. Srivastava, S. K. Tiwari, C. P. Singh, Phys. Rev. D 82, 014023 (2010).

[2] F. Brau and F. Buisseret, Phys. Rev. D 79, 114007 (2009).

[3] F. Giacosa, Phys. Rev. D 83, 114002 (2011).

[4] S. Borsanyi, et al., JHEP 11, 077 (2010).

[5] F. Karsch, Nucl. Phys. A 698, 199c-208c (2002).

[6] E. Shuryak, Prog. Part. Nucl. Phys. 62, 48 (2009).

[7] H. Satz, Int. J. Mod. Phys A 21, 672 (2006)

[8] E. V. Shuryak, Phys. Rep. 61, 71 (1980).

[9] J. Cleymans, R.V. Gavai, and E. Suhonen, ibid. 130, 217 (1986).

[10] P. Levai, U. Heinz, Phys. Rev. C 57, 1879 (1998).

[11] R. A. Schneider, W. Weise, Phys. Rev. C 64, 055201 (2001).

[12] A. Peshier, B. Kampfer, O.P. Pavlenko, G. Soff, Phys. Lett. B 337, 235 (1994).

[13] A. Peshier, B. Kampfer, O.P. Pavlenko, G. Soff, Phys. Rev. D 54, 2399 (1996).

[14] V. M. Bannur, Eur. Phys. J. C 50, 629 (2007).

[15] M. I. Gorenstein and S. N. Yang, Phys. Rev. D 52, 5206 (1995).

[16] V. M. Bannur, Phys. Lett. B 647, 271 (2007); J. Phys. G: Nucl. Part. Phys. 32, 993 (2006).

[17] V. Goloviznin and H. Satz, Z. Phys. C 57, 671 (1994). 
[18] S. S. Singh et al., Pram. J. Phys. 74, 27 (2010).

[19] Y. Kumar, JoP: Conf. Ser. 668, 012110 (2016).

[20] D. S. Gosain and S. S. Singh, Int. J. Theo. Phys. 53, 2688 (2014). 